\title{
ITERATED FAILURE RATE MONOTONICITY AND ORDERING RELATIONS WITHIN GAMMA AND WEIBULL DISTRIBUTIONS - CORRIGENDUM
}

\author{
Idir Arab and Paulo Eduardo Oliveira
}

doi:10.1017/S0269964817000481, Published by Cambridge University Press, 24 January 2018.

The original publication of this article [1] contained an error in the argument of the proof of Theorem 27, a key result for the criteria for stochastic ordering introduced. The correct proof is presented here.

The argument we used in the article for the proof of Theorem 27 below described the sign variation of $V_{s}(x)$ based on Lemma 26, which assumes that we are integrating a functions that does not depend on $x$. This is not the case for the representation considered. Therefore, we need to give another representation of $V_{s}(x)$ allowing to argue based on the referred lemma. This is given below, after recalling the statement of the result.

THEOREM 27: Let $X$ and $Y$ be random variables with absolutely continuous distributions with densities $f_{X}$ and $f_{Y}$, and distribution functions $F_{X}, F_{Y} \in \mathcal{F}$, respectively. If, for some positive integer $k \leq s$, and every $a>0$ and $b \in \mathbb{R}$, the function

$$
H_{k}(x)=\frac{1}{\prod_{j=1}^{k} \widetilde{\mu}_{Y, s-j}} \bar{T}_{Y, s-k}(x)-\frac{a^{k}}{\prod_{j=1}^{k} \widetilde{\mu}_{X, s-j}} \bar{T}_{X, s-k}(a x+b)
$$

changes sign at most twice, and if the change of signs occurs twice, it is in the order ",,+-+ ", as $x$ traverses from 0 to $+\infty$, then $F_{X} \leq_{s-I F R} F_{Y}$.

Proof: We rewrite $V_{s}(x)=\bar{T}_{Y, s}(x)-\bar{T}_{X, s}(a x+b)$ replacing successively the representation of each $\bar{T}_{Y, s}$ and $\bar{T}_{X, s}$ as an integral, according to (2), with a change of variables in the later case, we obtain the representation

$$
V_{s}(x)=\int_{x}^{\infty} \int_{x_{1}}^{\infty} \cdots \int_{x_{k-1}}^{\infty} H_{k}\left(x_{k}\right) d x_{k} \cdots d x_{2} d x_{1}
$$

Now, using iteratively Theorem 22 and Lemma 26 the proof is concluded.

(C) Cambridge University Press 2018 0269-9648/18 $\$ 25.00$ 
We need further to correct the expressions given after Remark 28 for the functions $H_{s}$ and $H_{s-1}$, introducing a factorial term:

$$
\frac{1}{(s-1) !} H_{s}(x)=\frac{1}{\mathbb{E} Y^{s-1}} f_{Y}(x)-\frac{a^{s}}{\mathbb{E} X^{s-1}} f_{X}(a x+b)
$$

and,

$$
\frac{1}{(s-1) !} H_{s-1}(x)=\frac{1}{\mathbb{E} Y^{s-1}} \bar{F}_{Y}(x)-\frac{a^{s-1}}{\mathbb{E} X^{s-1}} \bar{F}_{X}(a x+b) .
$$

Naturally, the factor $(s-1)$ ! has no influence on the sign variation, so Corollary 29 holds with exactly the same statement.

The authors regret these errors.

\section{Reference}

1. Arab, I. \& Oliveira, P.E. (2018). Iterated failure rate monotonicity and ordering relations within Gamma and Weibull distributions. Probability in the Engineering and Informational Sciences. doi:10.1017/S0269964817000481. 